This is the peer reviewed version of the following article: Helm, D. (2017) Can I Have A Word? Social Worker Interaction and Sense-Making. Child Abuse Rev., 26: 388-398, which has been published in final form at

https://doi.org/10.1002/car.2463. This article may be used for non-commercial purposes in accordance With Wiley Terms and Conditions for self-archiving. 


\title{
Can I Have A Word? Social Worker Interaction and Sense-Making
}

\author{
Duncan Helm, University of Stirling
}

\section{Introduction}

This paper considers data gathered from an ethnographic study of practice within a children and families social work office. The analysis of the collected data has been reported in a series of papers, each exploring specific aspects of social workers' sense-making. While research has provided new insights into social work assessment and decision-making, relatively little attention has been paid to the everyday practices of making sense of complex and ambiguous information. In particular, there is a dearth of research into the contribution which informal peer or colleague interaction makes to sense-making. This paper therefore seeks to shed further light on the role of informal interaction and discussion in sense-making.

Human reasoning is recognised to incorporate a blend of intuitive and analytical reasoning (Hammond et al. 1997). The properties of judgement tasks commonly encountered in social work (such as high levels of complexity and uncertainty) will provoke strongly intuitive forms of reasoning (Van der Luitgaarden 2009) but deliberate analytical reasoning is also required for abstraction, generalisation and more defensible decision-making (Munro 2008). Social workers therefore need to be able to move effectively between the poles of intuition and analysis.

Broadhurst et al. (2010) emphasised the centrality of social relations in social work practice, in the cultures of team and the creation of unique situations and also habitual responses. These everyday interactions have been steadily evolving and changing in response to new technologies and new working practices. In the midst of these changes, there is a risk that these 'seen but unnoticed' practices (Garfinkel 1967) have evaded proper scrutiny and consideration of their relevance to practice.

\section{Research design}

A small scale study was carried out in 2012 using an ethnographic approach to explore sense-making in a local authority children and families social work team. Access to the research site was negotiated in liaison with a Learning and Development Officer from the local authority. Ethical approval was gained from the University of Stirling Research Ethics Committee. Meetings with managers and team members provided opportunities to discuss and refine the methodology and ensure that participation was voluntary and based on informed consent. In the interests of confidentiality pseudonyms are used throughout the paper. Full details of ethical and methodological considerations are available in Author's own (2016)

The research site was a Children and Families Practice Social Work Team situated within a Scottish local authority. The team comprised 27 Social Workers, Senior Social Workers and Social Work Assistants, supervised by 7 Team Leaders under the overall management of a Practice Team Manager. The team occupied one floor of a building with a number of rooms of different sizes. This meant that room occupancy rates related to room size from singleoccupancy to multi-occupancy. 
Observations were carried out over twelve weeks with the researcher following the duty social worker in office-based work for a 4 hour period on the same day of the week over the period of study. Descriptive observation was used to collect data about the situation and nature of judgement and decision-making. Written recordings were made during observations allowing for data to be gathered across a range of dimensions and reviewed immediately to reduce known biases in attention and memory (Robson 2002)

Analysis of the data sought to explore the ways in which customs and practices within this office influenced and informed sense-making. Descriptive codes were developed initially from Hammond's (1996) model of the cognitive continuum, focusing on patterns and shifts between intuition and conscious reasoning in sense-making. As analysis progressed, I returned to memos (contemporaneous notes to myself on emerging ideas and views) and session summaries (drawn up at the end of each period of recording) to help generate connections and possible tentative explanations for the trends and patterns emerging from the data.

This was an exploratory study, aimed at describing and illustrating what can be argued to be the most inaccessible aspect of Pithouse's (1987) 'invisible trade' of social work; the process of sense-making. It is 'invisible' as it inherently intangible but it is also 'invisible' because it is so hard to consciously access and reflect upon our own sense-making activity. As such, an ethnographic approach has allowed me to describe some notable elements of the cultures and practices of sense making in the office studied. It has also supported the identification of themes and patterns which may support critical reflection in practice and inform further research.

\section{Findings}

I have selected three elements of sense-making which have emerged from the data as relevant and of interest to social work. Each theme will be introduced with some brief examples from the data and then illustrated in a fuller representative vignette. In the final section the findings will be considered in relation to the wider literature and significance for practice.

\section{"I'm really curious": Practising methodical doubt}

Practitioners in this study moved around their office space frequently, creating regular opportunities for informal case discussions. Within these discussions, practitioners seemed to avoid leaping to judgement through trusting their colleagues to tolerate their uncertainty and help them think things through.

\section{Examples}

- "my thinking is..."

- $\quad$ "I've covered the file but we don't really know much about them"

- "I was kind of like 'oh, that was quite odd!"'

Vignette 
Stephanie was the social worker on duty and was the sole occupant of a room with two desks. Lisa (colleague social worker) walked through from her own room and began the following conversation with Stephanie:

Lisa - "Can I have a word about the internet thing?"

Stephanie looked up and round to face Lisa who was standing at the door.

Lisa - "Gloria's taken Haley's phone off her"

Stephanie - "I don't know if she's taken into account how important a phone is to a teenage girl. Gloria's plan is to get her a "pay as you go" and pay the remainder of the contract costs out of the sale of the phone."

Lisa - "I know, it's about building up trust but the first thing she did was to go online with this man. She's fallen out with her best friend, Emma. We've got (counselling organisation currently involved) today so we'll see...they're good at reasoning things out with her. Haley can articulate to me but not her mum. See Karen (Haley's aunt)...Haley gets so much more out of her contact with her... Karen could do so much.

Stephanie - you'll need to check (client record system)...what age is her wee one? 3? 4?

Lisa moved into the room and sat on the edge of the spare desk

Lisa - I'm trying to remember. I'm really curious.... when you think about their relationship and then consider the difference between them. Karen's never used drugs or alcohol...maybe she's (Haley) on the autistic spectrum"

Stephanie - "mmh...something organic?"

Lisa - there's a lovely communication through the eyes

Stephanie - I've seen them at the supermarket. I live near them at (locality).

A striking feature of this exchange was the level of exploration, curiosity and hypothesis generation in the discussion. For Stephanie and Lisa, the focus was on constructing meaning rather than trying to identify solutions. Unlike the norms of line-managed supervision, there was a shared and detailed case knowledge but there was no note-taking nor expectation of problem-solving responses. Instead, both practitioners were able to be in the moment and engage fully with the uncertainty and complexity of this case scenario. The effect was to create a safe space which allowed the two workers to work methodically through doubt and explore a broad range of frames and perspectives.

\section{"Think about your role": proximity and reflexivity}

Face-to-face discussions happened as natural ongoing conversations in shared offices. This proximity over time appeared to provide opportunities for self-reflection. In this way, practitioners were supported to be more conscious and reflexive of their sense-making.

\section{Examples}

- "maybe I'm reading something into it..." 
- " we're terrified he's just going to slip under the radar"

- "reflecting back...perhaps I could have..."

Vignette

The duty social worker (Jack) and his colleague Rob were sitting at two desks facing each other. Jack was making a call to a father who has demanded a change of social worker (currently Rob). The father ended the call abruptly, leaving Jack to put down the receiver and look at Rob.

Rob (with empathy) "He's a difficult man".

Jack responded by repeating details of recent contacts and current concerns.

Rob; "I'm a little bit perturbed! I've got a really good relationship with these children and then an adult changes this..."

Jack: "...but you need to work with the parents to make the changes. People don't understand, you know, it's the Panel that makes the decision."

Rob: "I don't understand how they could hate someone so much (laughs) I'm the nicest guy in the world!"

Jack: "Think about your role and what you mean to them"

The shared space of this room allowed Rob to witness the call unobtrusively. As with the first example, the key elements of person and place facilitated the process of making sense of people's actions and about the motivations and beliefs underlying them. There was a shared case knowledge and a focus on curiosity and a search for possible explanations.

When Rob said "I'm a little bit perturbed", he did so with a slight smile and the kind of steadily rising pitch to indicate surprise. He expressed his concern that the adult had been able to have Rob removed as the children's supervising social worker. The sentence was unfinished and was spoken with a tone indicating difficult emotions of frustration and dissatisfaction. Jack's response provided Rob with an alternative explanation and a perspective which helped Rob to step back and process his emotions towards a more explicit and considered position.

\section{Information cocoons and shared resources}

This theme is perhaps more clearly illustrated with an extract from my field notes rather than by brief examples and a vignette. Sense-making activity is not restricted to dialogue and this extract offers some insight into how practitioners create and utilise artefacts and resources to support movement between intuitive and analytical modes of reasoning.

4 workers share this office. Each has a desk and a filing cabinet. There is also a small fridge with a kettle and coffee-making equipment on top. There are a range of photocopied and original documents on the filing cabinets, mainly guidance and procedural paperwork with 
some text books. On the walls there pictures of film stars, a large copy of the "my world triangle" (Scottish Government assessment tool), children's artwork and pictures of children.

The observation session begins as the office opens and following exchanges are observed in the first 20 minutes.

Caitlin has bought fresh pastries to share and Lisa is offering them to colleagues and the researcher. There is discussion with the new social worker, Natasha, about getting lost on a home visit. Caitlin asks for the address of a building. A colleague appears at the door asking for a printer code. Another colleague comes into the room to discuss a possible placement breakdown and Caitlin enquires "is that another case you are getting?" Lisa asks Natasha about her recent training on brain development. A team leader comes in to make a coffee; she has a pastry and discusses recent flooding with those nearby. Debbie speaks to Kim about text books which she has shared, indicating that she wishes to use Signs of Safety in assessment work with a child "doing the three houses". At this time, Kim is also accessing a web-based text on achieving better outcomes for looked-after children

Despite the move to digital media as a repository for knowledge, dialogue and hard copy still featured in the construction and use of professional knowledge. Knowledge of immediate practical application (such as directions and access codes) was also readily available. Individual practitioners have spent years weaving information cocoons around themselves, constituted of knowledge from their training, reading, experiences and reflections; providing a sense of continuity and order (Giddens 1991). Regular day-to-day routines helped these practitioners to penetrate these protective cocoons and share knowledge. Importantly, the range of resources was widened to include established and verifiable knowledge (such as published articles) and locally established and applied knowledge. This latter source provided crucial opportunities for dialogue and feedback on how research and theories can be applied to understand the unique circumstances of specific children and families.

Artefacts on the walls depicted practitioners' interests and identities; many of these artefacts related directly to matters of children and childhood. By publicly sharing artefacts, practitioners shared elements of their own personal and professional selves with colleagues. Some items (such as statements of children's rights or children's drawings) were displayed prominently in shared areas of the office whereas pictures of film stars or more personalised mementos from service users tended to be displayed so as to only be partially visible to others. This managed display of self can be seen as making values and attitudes explicit and providing those sharing the room with the opportunity to co-create identities which are mutually understood and revised over time.

\section{Discussion}

\section{Curiosity and practising methodical doubt}

In common with other studies (Jeyasingham 2015) direct face-to-face contact played a significant role in sense-making activity here. Practitioners actively sought out dialogue and appeared to make deliberate choices about who to speak to and when. In these situations, practitioners could be seen to create spaces within which it was possible to practice reflexivity and criticality. In this office, proximity was a key factor in the creation of thinking spaces; practitioners were more likely to have discussions with those closest to them. 
However, when more physically distant sources of colleague support were sought, this also brought greater levels of privacy. In this sense, practitioners benefitted from offices which offered flexibility and non-stigmatising choice in contacts and levels of privacy.

Practitioners in this study expressed a preference for face-to-face discussion with their managers because they were aware of the potential for "overload and miscommunication" in email contact. Practitioners were also able to have a different quality of dialogue with colleagues where the emphasis was less on decision-making and more on a deeper reflexive consideration of the data available. Broadhurst et al. (2010 p582) note that "Many social work tasks are difficult to achieve without face-to-face interaction..." and I would suggest here that analysis (sense making) is one of the tasks which could be more effectively supported through better use of face-to-face discussion where tacit knowledge is made explicit, where hypotheses are created and tested, and where curiosity and methodical doubt can be brought together to inform practice.

While this study has identified face-to-face communication as an important support to openminded critical thinking, it is notable that challenging dialogue and dialectic debate (Reder and Duncan 1999) was not strongly represented in the data. This suggests that informal discussion alone may not effectively guard against common biases of judgement and decision-making. Team values and cultures appeared to be highly influential in this study and this may be equally true for negative, limiting or unhelpful cultures. In this respect, there is a need to generate and support cultures which can tolerate uncertainty and value dissent and diversity.

\section{Proximity and reflexivity}

The kind of space provided by this office layout was significant to managing the complex emotional impact of child protection practice. Choice and opportunity for direct informal case discussion helped to manage and negotiate the boundary between the public and purposeful use of emotion and the private processing of emotions (Hochschild 1983). Practitioners in these offices had a culturally acceptable range of choices in relation to the time, person and content of these sense-making discussions. This created an environment where the practitioner themselves had a level of control over the boundary between public and private aspects of emotional work and sense-making. Proximity in this case supported timely feedback which can aid critical reflection right on the cusp between reflection-in-action and reflection-on-action. In this way, shared spaces create proximity and interaction that can support critical practice and provide a source of feedback.

Social work is a profession that requires combined skills in emotional and relationship-based work (Ruch 2007) and critical thinking. Sheppard and Charles (2014, p4) emphasise the connection between the two domains: "Social workers need to adopt critical thinking in their examination of the interpersonal...and require sensitivity and focus on the interpersonal for their critical thinking capabilities to serve any purpose in their conduct of practice." The vignettes in this study have illustrated how practitioners actively sought out and provided support and opportunities for critical evaluation of explicit and concrete knowledge and tacit embodied ways of knowing. However, performance management cultures can serve to reduce (even exclude) conversation with colleagues in pursuit of management targets (Wastell et al. 2010). Office spaces have the potential to support competing discourses and 
critical reflection but they also have the potential to simply reinforce dominant discourse and further reduce critical practice. As such, the resource of the shared space needs careful management and this paper seeks to encourage further debate about how organisational and professional values are generated and maintained through these spaces and resultant ways of sharing sense-making.

The office in this study offered proximity and choice for people seeking informal discussion due to the number of different rooms and people easily available. While there were significant instances of this aiding reflexivity, there is always the potential for practitioners to seek out colleagues who will provide familiarity and consent when it is perhaps novelty and dissent that are required. Self-selection and unquestioning adherence to group norms need to be guarded against and this is something that managers will consider in relation to supervision as well as creating diversity in room occupancy.

Many practitioners have indicated the benefits of engaging in emotional work with colleagues who know the reality of the situation and avoid the power imbalance which could exist in supervision. Studies have indicated that social workers have a preference for practice-based knowledge, conveyed from colleagues and experience, rarely consulting external sources (Avby et al. 2015). In this study, practitioners were actively engaging with external knowledge sources such as training, books and internet sources. However, a key element of this engagement with external sources was the level of dialogue within the team and this may indicate the role which informal interaction can play in supporting the update of evidence to inform practice.

\section{Secure base}

Many of the practices observed in this study seemed (on a psychosocial level) to be relevant to the development and maintenance of "secure base". Workers frequently shared food, made coffee for each other and moved from the room to room to make hot drinks. Food sharing can be seen as a means of communication (Counihan and Van Esterik 2013) and expressing emotion (Emond et al. 2014) with interactions supporting social cohesion through the demonstration of care, acts of reciprocity and the development of ritual and routine in the office. There is relatively little known about how these interactions are managed in practice (Gambrill 2012) but this study has identified the importance of trusting relationships in offices as a means of playing out conflicts and exploring hypotheses in a safe way. The development of trusting bonds or 'contracts' between workers in this office could be seen as foundational to sense-making, creating the secure base required to explore intuitive thinking and develop more structured analysis through movement to-and-fro on the cognitive continuum.

Social workers may prefer informal peer discussion to formal supervision as a forum for discussing emotional aspects of work (Ingram 2015). Within this study, informal discussion was the most prevalent forum for making sense of emotional information. Provided with a physical, ontological and emotional 'secure base' in their office and colleagues, practitioners were able to explore cognitively and emotionally in their analysis. Intellectually curious and professionally rigorous discussion was predicated upon a sense of secure base from which to begin to construct meaning. Openness, trust and collegiality can promote interconnected and critically reflexive practice (Ruch 2007) and this study highlighted the importance of 
office spaces in creating a safe environment for sense-making. If working practices and office spaces create a secure base for practitioners, it is more likely that they will be able to make use of colleagues to explore gut feelings and explicit reasoning with sufficient rigour and depth.

A key value on display here was a focus on the child, with many artefacts (such as artwork and images of childhood) celebrating the inherent worth and dignity which social workers ascribe to children and young people's lives and experiences. This is a value expressed in legislation and policy but one which practitioners struggle to apply consistently in decisionmaking. The use of these visual and emotionally connected artefacts was widespread in this office and worthy of consideration in relation to helping practitioners see the bigger picture and access embodied knowledge in their reasoning.

\section{Limitations of the study}

The non-participatory nature of data collection was part of measures to reduce bias by not influencing the judgements being made. I sought to minimise my impact through a range of other measures including use of doctoral supervision, attending team meetings, individual discussions with team members and careful consideration of positioning during recording periods. However, being observed does raise anxieties about being judged and findings may be affected by participants 'performing' while being observed (Punch 2005) or being so conscious of the researcher as to be unable to act and think naturally.

In data analysis, I sought out events and processes which provoked movement between intuitive and analytical modes of thinking. As such movement promotes good fit between the features of the judgement task and the form of thinking employed (Hammond 1996) this choice has resulted in findings which tend to focus on positives. While I have indicated the need for cautious interpretation of the findings, they must necessarily remain at quite a descriptive level. Ethnographies are difficult to replicate but these findings shine a light on a relatively under-researched element of social work practice and, as such, may help to inform debate in practice and guide further research.

\section{Conclusions and implications}

This research has identified some interesting facets of informal interaction in the social work office as an aid to sense-making. Rigorous judgement is at the heart of assessment practice and it is acknowledged that practitioners need support to move beyond gathering information to begin to make sense of what it means for their client's lived experience. In considering informal office-based interactions closely, it emerges that interactions with colleagues have the potential to offer the necessary support for the containment of emotions and development of knowledge. While this can offer a secure base from which to move out and explore a range of perceptions and hypotheses, there is potential for less desirable cultures to develop which may serve to exacerbate rather than counter bias. Practitioners should be aware of the influence of team cultures on sense-making and consider how they can make best use of their working environment to support effective judgement. While this study has identified the importance of informal discussion in sense-making, further research is needed to develop a fuller understanding of the implications for practice.

In this study, choice and access to a range of trusted colleagues was seen to promote critical thinking. This is in contrast to the situation that many social workers find themselves 
in where trust and security have been undermined by pressures such as high staff turnover, unmanageable caseloads and the rise of agile working practices. There is a need to consider the ways in which these factors interact and influence practice but it may be possible to draw on existing resources to develop teams as effective thinking units. This would require attention to the use of office space to deliver flexibly public/private spaces and the development of working cultures which view dialogue as a beneficial activity and one which is central to, as opposed to a barrier to, judgement and decision-making. Managers in particular may wish to consider ways of promoting working team cultures which facilitate challenging yet supportive dialogue as an aid to sense-making.

'Agile working' practices (such as hot-desking and remote working) are rapidly expanding in social work teams across the UK. The benefits of such practices are largely seen in reduced costs and more flexible use of office space (Kim and deDear 2013). However, the messages from this study indicate that agile working practices may also inflict costs because there are fewer opportunities to build trusting relationships and have the informal discussions that have been shown to support methodical doubt in practice. Curiosity, rigorous thinking and reflexivity are important elements of effective, child-centred social work and these findings suggest a need to think carefully about the consequences of agile practices for professional thinking. Managers may consider adopting ethnographic approaches as a means of evaluating and gaining a fuller understanding of the consequences of agile working practices on sense-making.

Models already exist offering strategic development of cross-team contact to aid judgement. The Reclaiming Social Work model (Trowler and Goodman 2011) is currently one of the more clearly defined approaches and has received some positive evaluation (Forrester et al. 2013). It is noticeable that the two key features of the Reclaiming Social Work model (shared work and the quantity and quality of case discussion) have also been identified in this study as facilitators for effective analysis within traditional organisational structures. Within existing models of delivery there are underutilised resources in many teams and it may be possible to release these resources by fine-tuning the way in which colleagues collaborate in sensemaking rather than a wholesale, expensive and potentially disruptive redesign of the service.

\section{Key practitioner messages}

- curiosity and methodical doubt are central elements in effective social work sensemaking

- social workers can use informal discussions effectively to support open-minded and rigorous sense-making

- choice and proximity of colleagues can promote shared sense-making

- The nature of office spaces may influence the sense of self security that underpins effective sense-making

\section{References}

Avby, G., Nilsen, P. and Ellström, P. (2015) Knowledge use and learning in everyday social work practice: a study in child investigation work. Child \& Family Social Work. [online resource] http://dx.doi.org/10.1111/cfs.12227 last accessed 22.07.15

Broadhurst, K., Hall, C., Wastell, D., White, S. and Pithouse, A. (2010) Risk, Instrumentalism and the Humane Project in Social Work: Identifying the Informal Logics of Risk Management 
in Children's Statutory Services British Journal of Social Work first published online February 1, 2010 doi:10.1093/bjsw/bcq011

Counihan, C. and Van Esterik, P. (2013) Food and Culture: A Reader. (3rd edition). Abingdon: Routledge.

Emond, R., Mclntosh, I. and Punch, S. (2014) Food and Feelings in Residential Childcare. British Journal of Social Work. 44 (7) pp1840-1856.

Forrester, D., Westlake, D., McCann, M., Thurnham, A., Shefer, G., Glynn, G. and Killian, M. (2013) Reclaiming Social Work? An Evaluation of Systemic Units as an Approach to Delivering Children's Services Summary report of a comparative study of practice and the factors shaping it in three local authorities [online resource] https://www.beds.ac.uk/_data/assets/pdf_file/0008/256742/Short-Systemic-UnitReport-June-2013.pdf last accessed 23.07.15

Gambrill, E. (2012) Critical Thinking in Clinical Practice. 3rd Edition. New Jersey: Wiley and Sons.

Garfinkel, H. (1967) Studies in Ethnomethodology. Englewood Cliffs, NJ: Prentice-Hall.

Giddens, A. (1991) Modernity and Self-Identity: Self and Society in the Late Modern Age. Cambridge: Polity Press.

Hammond, K. (1996) Human Judgement and Social Policy: Irreducible Uncertainty, Inevitable Error and Unavoidable Injustice. Oxford: Oxford University Press.

Hammond, K., Hamm, R., Grassia, J. and Pearson, T. (1997) 'Direct comparison of the efficacy of intuitive and analytical cognition in expert judgement.' In Goldstein, W. and Hogarth, R. (Eds) (1997) Research on Judgement and Decision Making. Cambridge: Cambridge University Press.

Author's own (2016)

Hochschild, A. (1983) The Managed Heart. California: University of California

Ingram, R. (2015) Exploring Emotions within Formal and Informal Forums: Messages from Social Work Practitioners. British Journal of Social Work. 45 (3) pp896-913 first published online October 14, 2013 doi:10.1093/bjsw/bct166 accessed 15.05.15

Jeyasingham, D. (2015) Open spaces, supple bodies? Considering the impact of agile working on social work office practices Child \& Family Social Work [online resource]http://dx.doi.org/10.1111/cfs.12130 last accessed 22.07.15

Kim, J. and deDear, R. (2013) Workspace satisfaction: The privacy-communication trade-off in open-plan spaces. Journal of Environmental Psychology 36 pp18-26

Munro, E. (2008) Effective Child Protection. 2nd Ed. London: Sage.

Pithouse, A. (1987) Social Work: the social organisation of an invisible trade. Aldershot: Avebury. 
Punch, K. (2005) Introduction to Social Research: Quantitative and Qualitative Approaches. (2nd Ed). London: Sage.

Reder, P. and Duncan, S. (1999) Lost Innocents: A follow-up study of fatal child abuse. Hove: Routledge.

Robson, C. (2002) Real World Research. Oxford: Blackwell.

Ruch, G. (2007) 'Thoughtful' practice: childcare social work and the role of case discussion. Child Abuse Review. http://onlinelibrary.wiley.com.ezproxy.stir.ac.uk/doi/10.1111/j.13652206.2006.00466.x/epdf last accessed 21.07.15

Sheppard, M. and Charles, M. (2014) Head and Heart: An Examination of the Relationship Between the Intellectual and Interpersonal in Social Work. British Journal of Social Work. Online Advance access published November 42014

Trowler, I. and Goodman, S. (Eds) (2012) Social Work Reclaimed: Innovative Frameworks for Child and Family Social Work Practice. London: Jessica Kingsley.

Turney, D., Platt, D., Selwyn, J. and Farmer, E. (2012) Improving Child and Family Assessments: Turning Research into Practice. London: Jessica Kingsley.

Van de Luitgaarden, G. (2009) Evidence-Based Practice in Social Work: Lessons from Judgement and Decision-Making Theory. British Journal of Social Work. 39, pp243-260

Wastell, D., White, S., Broadhurst, K., Peckover, S. and Pithouse, A. (2010) Children's services in the iron cage of performance management: street-level bureaucracy and the spectre of Švejkism. International Journal of Social Welfare. 19 pp310-320 doi:10.1111/j.1468-2397.2009.00716.x 